Please do not remove this page

RMIT

UNIVERSITY

\title{
Performance of quadratic time-frequency distributions in blind source separation of speech signals
}

Shang, Lei; Hussain, Zahir; Harris, Richard

https://researchrepository.rmit.edu.au/esploro/outputs/9921857913201341/filesAndLinks?institution=61RMIT_INST\&index=null

Shang, L., Hussain, Z., \& Harris, R. (2004). Performance of quadratic time-frequency distributions in blind source separation of speech signals. Proceedings of the 2004 IEEE International Symposium on Spread Spectrum Techniques and Applications, 905-908.

https://researchrepository.rmit.edu.au/discovery/fulldisplay/alma9921857913201341/61RMIT_INST:Resea rchRepository

Repository homepage: https://researchrepository.rmit.edu.au

(c) 2004 IEEE. Personal use of this material is permitted. However, permission to reprint/republish this material for advertising or promotional purposes or for creating new collective works for resale or redistribution to servers or lists, or to reuse any copyrighted component of this work in other works must be obtained from the IEEE.

Downloaded On 2023/04/26 16:43:59 +1000 


\title{
PERFORMANCE OF QUADRATIC TIME-FREQUENCY DISTRIBUTIONS IN BLIND SOURCE SEPARATION OF SPEECH SIGNALS
}

\author{
Lei Shang, Zahir M. Hussain and Richard Harris \\ Centre for Advanced Technology in Telecommunications \\ School of Electrical and Computer Engineering \\ RMIT University, Melbourne, Victoria, Australia. \\ Email: s9612136@student.rmit.edu.au,zmhussain@ieee.org, richard@catt.rmit.edu.au
}

\begin{abstract}
Blind source separation (BSS) based on time-frequency distributions (TFD's) has certain advantages over other methods, such as allowing the separation of Gaussian sources with identical spectral shape but different $t$ - $f$ localization properties. However, quadratic TFD's, which are the most important class of TFD's suitable for this approach, differ widely in resolution and ability to reduce the cross-terms that disturb the signal interpretation. This paper investigates the performance of different time-frequency distributions on the blind separation of speech signals and non-linear FM signals. We also discuss the possibility and the suitability of the solution to convolutive mixture of the speech signals using time-frequency based blind source saparation because of its ability to process complex-valued signals.
\end{abstract}

\section{INTRODUCTION}

Blind Source Separation (BSS) has wide applications in different fields such as communication, image processing, etc [1]. BSS is used to recover the original waveforms of sources without a prior knowledge of the mixture's structure. The mixture in BSS can be convolutive or instantaneously linear. A time-frequency based BSS approach was presented in [2] to solve instantaneous mixture problem. TFD's reveal information about the multicomponent nature of signals that other means cannot reveal. In this paper, we investigate the performance of the above approach using the optimal smoothing kernels designed in [3]. At the end of the paper, we discuss the suitability and possibility of applying the time-frequency based BSS to convolutive mixture.

A subclass of Cohen's Class of time-frequency distributions, referred to as the T-distributions, was presented in [3] and shown to be suitable for efficient amplitude and instantaneous frequency (IF) estimation of mono- and multicomponent FM signals. The exponential and hyperbolic timeonly kernels have proven to be efficient in reducing crossterms while retaining high resolution, with a compromise between these two requirements depending on the selected parameters. In this paper, we apply these kernels to timefrequency based blind source separation of speech signals, linear FMs and non-linear FMs to investigate possible performance improvements based on optimal kernel selection. The simulation results will show that the T-distributions have advantages over Wigner-Ville and Choi-William distributions in terms of noise performance, stability, and wider optimal dynamic range.

\section{METHOD DESCRIPTION}

We assume that the multidimensional observations of the signal $\mathbf{s}(t)$ are given by

$$
\mathbf{x}(t)=\mathbf{y}(t)+\mathbf{n}(t)=\mathbf{A} \mathbf{s}(t)+\mathbf{n}(t)
$$

where $\mathbf{x}(t)=\left[x_{1}(t), \ldots x_{n}(t)\right]^{T}$ is the observed noisy instantaneous linear mixture of source signals given by $\mathbf{s}(t)=$ $\left[s_{1}(t), \ldots s_{n}(t)\right]^{T}$ and $\mathbf{n}(t)$ is the additive noise. The $m \times n$ matrix $\mathbf{A}$ is the mixing matrix. The assumptions here are that the source signal vector $\mathbf{s}(t)$ is a non-stationary multivariate process with its components mutually uncorrelated, with their cross-correlation equal to zero. The additive noise $\mathbf{n}(t)$ is a stationary, temporally white, zero-mean, complex random process, independent of the source signals.

In addition to the above assumptions, we also take advantage of the indeterminacies in the sources by normalizing the source signals to unit power; this implies that the covariance matrix $\mathbf{R}_{s}$ of $\mathbf{s}(t)$ is the identity matrix, that is, $\mathbf{R}_{s}=\mathbf{I}$, so that the covariance matrix $\mathbf{R}_{y}$ of $\mathbf{y}(t)$ is

$$
\mathbf{R}_{y} \stackrel{\text { def }}{=} \lim _{T \rightarrow \infty} \sum_{t=1}^{T} y(t) y^{*}(t)=\mathbf{A} \mathbf{A}^{H}
$$

where the superscript ${ }^{H}$ denotes the complex conjugate transpose of a matrix, and ${ }^{*}$ denotes the conjugate transpose of a vector. 
The method is a two-step process involving whitening and diagonalizing schemes as presented below:

Whitening: to transform the mixing matrix $\mathbf{A}$ into a unitary matrix. A whitening matrix $\mathbf{W}$ is applied to the observed $\mathbf{x}(t)$ so that

$$
\mathbf{z}(t) \stackrel{\text { def }}{=} \mathbf{W} \mathbf{x}(t)=\mathbf{W}(\mathbf{A s}(t)+\mathbf{n}(t))=\mathbf{U} \mathbf{s}(t)+\mathbf{W} \mathbf{n}(t))
$$

The matrix $\mathbf{U}$ is a unitary matrix, and the matrix $\mathbf{W}$ can be estimated by the following implementation:

1. Estimate the autocorrelation matrix $\hat{\mathbf{R}}$ from data samples. Denote by $\lambda_{1}, \ldots \lambda_{n}$ the $n$ largest eigenvalues and $h_{1}, \ldots, h_{n}$ the corresponding eigenvectors.

2. Under the white noise assumption, an estimate of the noise variance is the average of the smallest eigenvalues of $\hat{\mathbf{R}}$.

We then have the estimation of $\mathbf{W}$ as

$$
\hat{\mathbf{W}}(t)=\left[\left(\lambda_{1}-\hat{\sigma}^{2}\right)^{-\frac{1}{2}} h_{1}, \ldots\left(\lambda_{n}-\hat{\sigma}^{2}\right)^{-\frac{1}{2}} h_{n}\right]^{H} .
$$

Diagonalizing: to jointly diagonalize a set of data-STFD (spatial $t$ - $f$ distribution) matrices and retrieve the unitary matrix $\mathbf{U}$. The STFD matrix is $\left[\mathbf{D}_{x x}(t, f)\right]_{i j}=D_{x_{i} y_{j}}(t, f)$ for $i, j=1, \ldots n$, where $D_{x_{i} y_{j}}(t, f)$ is the discrete-time form of the Cohen's class of TFD's, given by [4]

$$
\begin{aligned}
D_{x_{i} y_{j}}(t, f)= & \sum_{l=-\infty}^{\infty} \sum_{m=-\infty}^{\infty} \phi(m, l) x_{i}(t+m+l) \\
& \times x_{j}^{*}(t+m-l) e^{-j 4 \pi f l}
\end{aligned}
$$

The matrix $\mathbf{U}$ can then be estimated by the following implementation:

1. Form $K$ matrices by computing the STFD of $z(t)$ for a fixed set of $\left(t_{i}, f_{i}\right)$ points, $i=1, \ldots, K$, corresponding to signal auto-terms.

2. A unitary matrix is then obtained as a joint diagonalizer of the set $\left\{D_{z z}\left(t_{i}, f_{i}\right) \mid i=1, \ldots K\right\}$

The obtained $\hat{\mathbf{U}}$ and $\hat{\mathbf{W}}$ from the above two steps can then be used to estimate the source signal $s(t)$ and mixing matrix $\mathbf{A}$ as: $\hat{s}(t)=\hat{\mathbf{U}}^{H} \hat{\mathbf{W}} x(t)$, and $\hat{A}=\hat{\mathbf{W}}^{\#} \hat{\mathbf{U}}$, where the superscript \# denotes the Moore-Penrose pseudoinverse.

The performance index is the interference-to-signal ratio (ISR), defined as [2]:

$$
\mathcal{I}_{p q}=E\left|\left(\hat{\mathbf{A}}^{\#} \mathbf{A}\right)_{p q}\right|^{2} .
$$

$I_{p q}$ measures the ratio of the power of the interference of the $q^{t h}$ source to the power of the $p^{t h}$ source signal estimated as in (6), and the global rejection level is defined as

$$
\mathcal{I}_{\text {perf }} \stackrel{\text { def }}{=} \sum_{q \neq p} I_{p q}
$$

\section{SELECTED TFD'S}

As mentioned in the previous section, a TFD that provides a good reduction of the cross-terms is needed to make sure that the off-diagonal elements of the TFD matrix of the sources are negligible and so that a diagonal structure can be maintained.

The time-frequency distribution of the analytic signal $z(t)$ associated with the original real signal $s(t)$ can be expressed as follows [3]

$$
\rho(t, f)=\underset{\tau \rightarrow f}{\mathcal{F}}\left[\phi(t, \tau) \underset{(t)}{*} K_{z}(t, \tau)\right]
$$

where $K_{z}(t, \tau)=z\left(t+\frac{\tau}{2}\right) z\left(t-\frac{\tau}{2}\right)$ is the instantaneous autocorrelation product, $\mathcal{F}$ is the Fourier transform, and $*$ denotes time convolution. The time-lag kernel $\phi(t, \tau)$ completely characterizes the corresponding TFD.

Wigner-Ville distribution (WVD) and Choi-Williams distribution (CWD) are the most famous TFDs of Cohen's Class [4]. Time-only kernels (kernels of the T-distributions) presented in [3] were shown to be more efficient than their twodimensional counterparts in Cohen's Class in terms of $t$ - $f$ resolution and cross-terms reduction, thus potentially they are more efficient in time-frequency based blind source separation. In this work we consider the above TFDs with the following kernels:

1. The constant kernel $\phi(t, \tau)=\delta(t)$, which corresponds to the Wigner-Ville distribution (WVD).

2. The exponential kernel of the Choi-Williams distribution(CWD), defined by

$$
\phi(t, \tau)=\sqrt{\alpha / 4 \pi \tau^{2}} e^{-\alpha t^{2} / 4 \tau^{2}}
$$

where $\alpha$ is a real positive parameter.

3. The exponential time-only kernel, defined by

$$
\phi(t, \tau)=\phi_{\alpha}(t)=\sqrt{\alpha / \pi} e^{-\alpha t^{2}}
$$

where $\alpha$ is a real parameter and $\sqrt{\alpha / \pi}$ is the normalization factor.

4. The hyperbolic time-only kernel, defined by

$$
\phi(t, \tau)=\phi_{\alpha}(t)=k_{\alpha} / \cosh ^{2 \alpha}(t)
$$

where $\alpha$ is a real positive number and the normalization factor $k_{\alpha}=\int_{-\infty}^{\infty} 1 / \cosh ^{2 \alpha} d t$ which equals $\Gamma(2 \alpha) / 2^{2 \alpha-1} \Gamma^{2}(\alpha)$ [ $\Gamma$ is the gamma function]. 


\section{NUMERICAL SIMULATION}

To investigate the potential performance improvements of the T-distributions, we carried out numerical simulations as shown in the following examples.

Example 1: The source signals are two speech signals, with sample size $N=512$. The $3 \times 2$ complex mixing matrix is arbitrarily chosen using the Matlab expression: $\mathbf{A}=\operatorname{rand}(3,2)+j * \operatorname{rand}(3,2)$.

The hyperbolic distribution is used to separate these two signals. Fig. 1 and Fig.2 illustrate the successful separation in the time domain and time- frequency distribution, respectively. Note that there are three mixed signals since the number of sensors is three (the third one is not plotted here). Notice the reverse order of the estimated signals. This permutation (more obvious with $n>2$ ) is caused by the inability to determine the ordering and the phases of the mixture matrix $\mathbf{A}$, hence any permutation of the estimated sources is also a satisfactory solution. We can also notice the phase shift of the recovered signals since the mixing matrix $\mathbf{A}$ is a complex matrix.

Example 2: The source signals are two non-linear FM (parabolic law) signals with total length $N=512$ and instantaneous frequencies given according to:

$$
s(t)=e^{j 2 \pi\left(A_{0} t+\frac{A_{1}}{2} t^{2}+\frac{A_{2}}{3} t^{3}\right)} .
$$

The $3 \times 2$ complex mixing matrix is arbitrarily chosen using Matlab expression: $\mathbf{A}=\operatorname{rand}(3,2)+j * \operatorname{rand}(3,2)$. This time the exponential distribution is used to separate these two signals. The separation process is similar to that shown in Fig. 1 and Fig. 2. For space limitations, we do not show the time-frequency distributions of the above signals.

All the four distributions (mentioned above) are used to separate these two non-linear FMs to compare the performance. Fig. 3 shows the mean rejection level versus different values of $\alpha$ for the above-mentioned four kernels. These two non-linear FMs are close enough together that the Wigner-Wille distribution has failed to separate them because of the large cross-terms.

It can be concluded that the two T-distributions provide a wider range of optimal values. The simulation also shows that the T-distributions are more stable and less signal-dependent in comparison to Wigner-Wille and Choi-Williams distributions. Fig. 3 also demonstrates the fact that the two Tdistributions converge to the Wigner-Wille case as $\alpha$ becomes large. This is in accord with the fact that as $\alpha$ increases, the T-distributions converge to WVD [5].

The optimal parameter value $\alpha_{\text {opt }}$ (in terms of the mean rejection level) for each TFD is then obtained from Fig. 3. For Choi-Williams distribution, $\alpha_{\text {opt }}=0.05$, for the hyperbolic T-distribution $\alpha_{\mathrm{opt}}=0.15$, and for the exponential T-distributions, $\alpha_{\text {opt }}=0.3$. We then obtain the noise performance of the different TFDs as shown in Fig. 4. The mean rejection levels are evaluated here over 100 Monte-
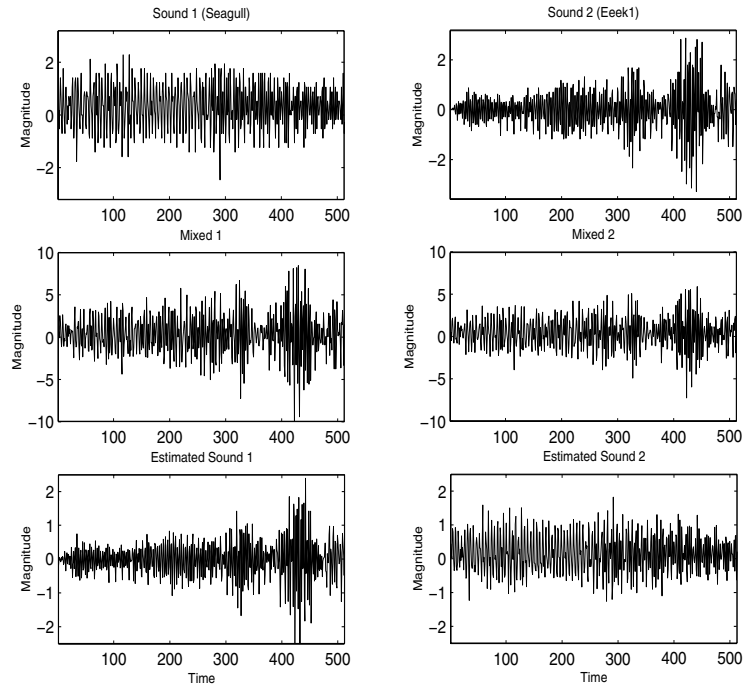

Fig. 1. Time-domain signals used for blind source separation with the hyperbolic T-distribution $(\mathrm{SNR}=50 \mathrm{~dB})$. First row: two test sound signals. Middle row: signals resulting from mixing the above sound signals using a randomly-generated mixing matrix. Last row: blindly separated two sound signals (compare with the first row).

Carlo runs. The performance improvement using time-only kernels is clearly observed in Fig. 4.

\section{CONVOLUTIVE MIXTURES}

We consider the following signal model for convolutive mixture. Let $\mathbf{s}(n)=\left[s_{1}(n), \ldots s_{N}(n)\right]^{T}$ be the vector of $N$ sources whose exact probability density functions are unknown. We assume that the sources are real-valued, nonGaussian distributed and statistically independent. For $M$ sensors, the observed signal vector $\mathbf{x}(n)=\left[x_{1}(n), \ldots x_{M}(n)\right]^{T}$ can be expressed as a convolutive combination:

$$
\mathbf{x}(n)=\sum_{k=-\infty}^{\infty} \mathbf{A}(k) \mathbf{s}(n-k)
$$

where $\mathbf{A}(k)$ is an unknown $M \times N$ matrix representing the mixing system. A convolutive mixture in the time domain corresponds to an instantaneous mixture in the frequency domain. In the frequency-domain, the convolutive mixture (12) takes the form

$$
\mathbf{X}(\omega)=\mathbf{A}(\omega) \mathbf{s}(\omega)
$$

where $\mathbf{X}(\omega), \mathbf{s}(\omega)$ and the matrix $\mathbf{A}(\omega)$ represent the observations, the sources and the mixing coefficients in the frequency - domain, respectively. We notice a resemblance of (13) to (1). That is, the frequency elements we observe 

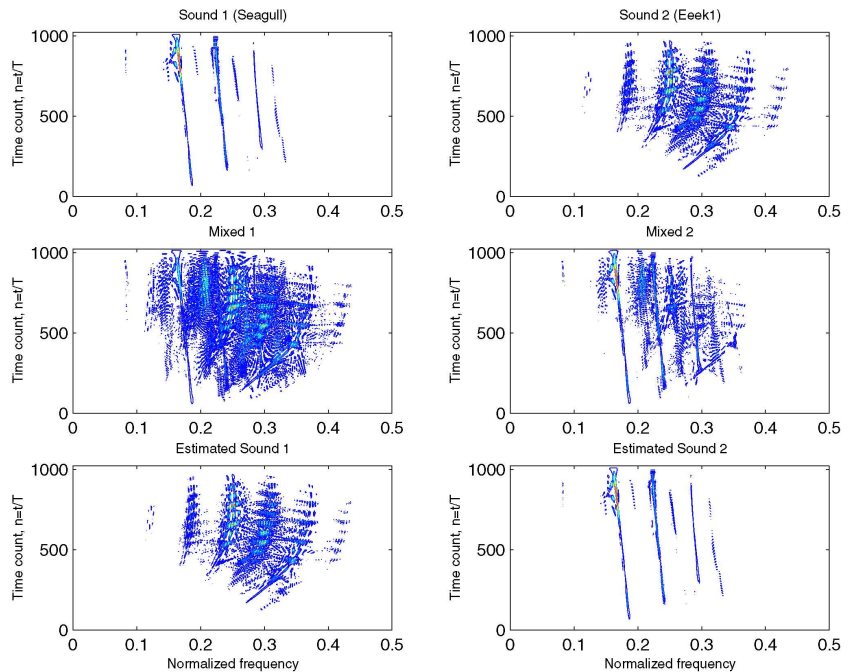

Fig. 2. Hyperbolic T-distribution of the signals shown in Fig.(1) used in blind source separation process. The obtained mean rejection level (MRL) is $-23.8 \mathrm{~dB}$.

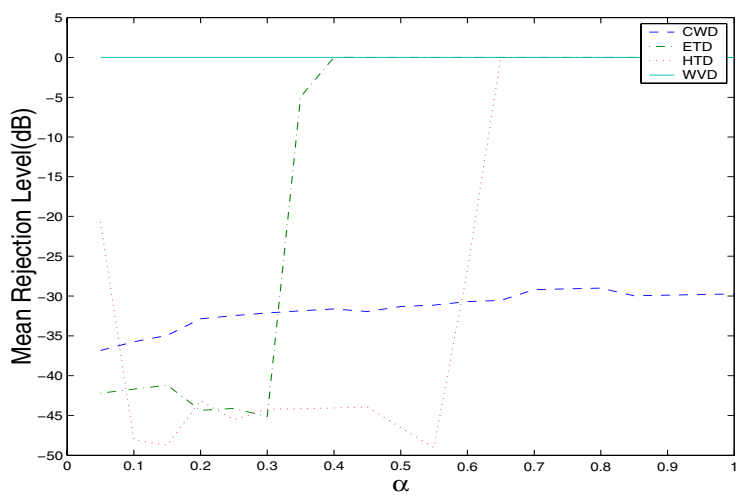

Fig. 3. Performance of different TFD's versus their parameter $\alpha$ in terms of the mean rejection level.

from our sensors are in fact instantaneous mixtures of the original frequency elements of the sources. Time - frequency based BSS separates complexed - valued source signal. Thus the separation of convolutive mixture can be at least theoretically achieved using the time - frequency based BSS.

\section{CONCLUSIONS}

A comparative study has been done using some well-known and recently-proposed TFDs.

It is shown that the choice of the time-frequency distribution (TFD) has a direct impact on the performance of $t-f$ based blind source separation (BSS). It is also true that the

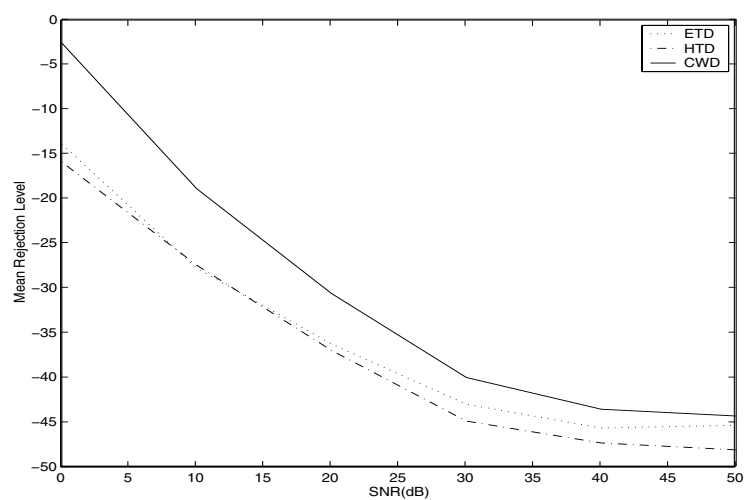

Fig. 4. Performance of different TFD's versus SNR.

$t-f$ based methods is highly signal-dependent.

It is found that the T-distributions (TFDs with time-only kernels) have advantages over Choi - Williams and Wigner - Ville distributions in the above $t$ - $f$ based BSS: they have wider and more stable optimal ranges (less signal-dependent); also better noise performance than WVD or CWD for the separation of non-linear FMs.

The optimal value of $\alpha$ is signal-dependent and is obtained by sweeping through all possible values by computer simulations. For the T-distributions the optimal values are easier to obtain than for Choi-Williams distribution.

\section{REFERENCES}

[1] A. Hyvarinen, J. Karhunen, and E. Oja, Independent Component Analysis, John Wiley \& Sons, 2001.

[2] A. Belouchrani and M. G. Amin, "Blind source separation based on time-frequency signal representations," IEEE Trans. Signal Processing, vol. 46, no. 11, pp. 2888-2897, Nov. 1998.

[3] Z. M. Hussain and B. Boashash, "Design of timefrequency distributions for amplitude and IF estimation of multicomponent signals," The Sixth International Symposium on Signal Processing and Its Applications (ISSPA’2001), 13-16 Aug. 2001.

[4] L. Cohen, Time-Frequency Analysis, Prentice-Hall, Englewood Cliffs, NJ, 1995.

[5] Z. M. Hussain and B. Boashash, "Adaptive instatntaneous frequency estimation of multicompoenent FM signals using time-frequency distributions," IEEE Trans. Signal Processing, vol. 50 , no. 8, pp. 1866 1876, Aug. 2002. 